\title{
Gleichzeitige Bestimmung der Aktivität von Acetylcholin-Esterase (EC 3.1.1.7) in Vollblut, Plasma und Erythrocyten mit dem automatischen Titrator
}

\author{
Von M. NENNER \\ Institut für Pharmakologie und Toxikologie der Universität Göttingen
}

(Eingegangen am 22. Juni 1970)

\begin{abstract}
Die Verwendung eines automatischen Titrators ermöglicht die rasche und zuverlässige Bestimmung der Acetylcholin-Esterase-Aktivität für Vollblut, Plasma und Erythrocyten.

Im Gegensatz zu anderen Methoden wird die Acetylcholin-Esterase-Aktivität einer Vollblutverdünnung und die eines daraus gewonnenen erythrocytenfreien Überstandes bestimmt. Dadurch werden Ungenauigkeiten beim Abtrennen des Plasmas, Zeitverluste durch mehrmaliges Waschen und Zentrifugieren der Erythrozyten und Pipettierfehler bei der Zugabe der Proben weitgehend ausgeschaltet.

Die Aktivitätsbestimmung wird bei pH 7,4 und $37^{\circ}$ in Gegenwart von 3mM Acetylcholin durchgeführt. Für den Standardansatz (Doppelbestimmung) werden $2 \mathrm{~m} /$ Blut benötigt. Eine Reduzierung dieser Menge ist möglich. Aus der Aktivitätsbestimmung für Vollblutverdünnung und Uberstand läßt sich bei Kenntnis des Hämatokritwertes die Acetylcholin-Esterase-Aktivität für Vollblut, Plasma und Erythrocyten berechnen. Die Angabe erfolgt in $\mu \mathrm{Mol}$ Acetylcholin $\mathrm{m}^{-1} \mathrm{Min}^{-1}$.
\end{abstract}

Für ein Kollektiv von 12 Männern und 8 Frauen wurden die Normalwerte bestimmt.

Simultaneous determination of the acetylcholinesterase activity of whole blood, plasma and erytbrocytes with the automatic titrator

Automatic titration allows a rapid and precise determination of acetylcholinesterase activity in whole blood, plasma and erythrocytes. In contrast with other methods, acetylcholinesterase activity is determined in diluted whole blood and in an erythrocyte-free supernatant prepared from the same dilution. In this way difficulties in the separation of plasma, loss of time by repeated washing and centrifuging of the erythrocytes and errors in sampling are greatly diminished. Activity is determined at $\mathrm{pH} 7.4$ and $37^{\circ} \mathrm{C}$ in the presence of $3 \mathrm{mM}$ acetylcholine. The standard test (performed in duplicate) uses $2 \mathrm{ml}$ of blood. This volume may be reduced. From the determination of the activity of diluted whole blood and the supernatant the acetylcholinesterase activity of whole blood, plasma and erythrocytes is calculated by use of the hematocrit value. Activity is given in $\mu$ moles acetylcholine $\mathrm{m}^{-1} \mathrm{~min}^{-1}$.

Normal values are given for a group of 12 men and 8 women.

Für die Diagnose und besonders zur initialen Kontrolle des Therapie-Erfolgs bei Organophosphatvergiftungen gewinnt die Bestimmüng der Aktivität der Acetylcholin-Esterase (EC 3.1.1.7) im Blut bzw. in den Erythrocyten immer mehr an Bedeutung, da sie im Gegensatz zum Verhalten der Plasma-Cholinesterase (EC 3.1.1.8) eine vertretbare Korrelation zum Vergiftungszustand zeigt $(1,2,3)$.

Für die klinische Routine-Untersuchưng sind die bisher üblichen Methoden zur Bestimmung der Acetylcholinesterase-Aktivität wenig geeignet.

Bei der manometrischen Methode $(4,5)$ wird das durch die enzymatisch gebildete Essigsäure freigesetzte $\mathrm{CO}_{2}$ bestimmt. Die Methode hat den Vorteil, daß 12 Proben gleichzeitig gemessen werden können. Thre Nachteile sind lange Vorbereitungs- und Meßzeiten, hohe Störanfälligkeit und die Verwendung einer Substratkonzentration von $20,6 \mathrm{~mm}$ Acetylcholin, bei der bereits eine beträchtliche Substrathemmung vorliegt. Die Variationsmöglichkeiten der Versuchsbedingungen sind geting.

Die potentiometrische Methode nach Mrchex (6) arbeitet mit dem geringsten apparativen Aufwand, hat aber den Nachteil, daß nicht bei konstantem pH-Wert gemessen wird. Außerdem handelt es sich um eine $Z$ weipunkt-Messung mit langer Inkubationszeit, deren Ergebnis $(\Delta \mathrm{pH} / \mathrm{Std}$.) keine Umrechnung in molare Umsatzgrößen erlaubt. Die Meßergebnisse können folglich nicht mit denen anderer Methoden verglichen werden.

Bei den photometrischen Methoden wird die Abnahme des Substrats (7) oder die Reaktion eines Produktes der enzymatișchen Hydrolyse mit einem spezifischen Reagenz $(8,9)$ gemessen. Diese photo- metrischen Methoden arbeiten rasch und zuverlässig, sind aber in der Wahl der Substrate begrenzt und auf Lösungen mit störender Eigenabsorption (Erythrocytensuspensionen, Hämolysate) nicht anwendbar.

Bei der potentiometrischen Titration $(10,11)$ wird der $\mathrm{pH}$-Wert im Testansatz durch kontinuierliche Titration mit Natronlauge konstant gehalten (pH-StatMethode). Die in der Zeiteinheit benötigte Menge ist der enzymatisch gebildeten Essigsäure und damit dem Substratumsatz proportional. Diese Methode arbeitet rasch und zuverlässig. Ihr Nachteil ist, daß jeweils nur eine Probe gemessen werden kann. Für kinetische Messungen hat diese Methode gegenüber den oben genannten jedoch beträchtliche Vorteile, da sie eine sehr weitgehende Variation von $\mathrm{pH}$-Wert, Temperatur, Substrat, Salzen und Inhibitoren erlaubt und eine Zugabe oder Entnahme von Proben jederzeit möglich ist.

Die nachfolgend vorgeschlagene Methodik hat für das klinische Laboratorium besonders in Vergiftungszentren den Vorteil, daß ein zuverlässiges Ergebnis innerhalb von 30 Minuten vorliegt.

\section{Prinzip}

Die hier beschriebene Methode erlaubt bei geringem Arbeitsaufwand (Titration von 2 Proben, Bestimmung des Hämatokritwertes) die gleichzeitige Bestimmung 
der Acetylcholinesterase-Aktivität in Vollblut, Plasma und Erythrocyten. Um die bei kleinen Blutvolumina schwierige Auftrennung in Plasma und Erythrocyten und die Zeitverluste beim mehrmaligen Waschen und Zentrifugieren der Erythrocyten zu vermeiden, wird die Blutprobe vor dem Zentrifugieren 1:50 verdünnt. Bestimmt man anschließend die Aktivität für einen aliquoten Anteil der Vollblutverdünnung ( $5 \mathrm{ml} \triangleq 100 \mu l$ Vollblut) und für den nach dem Zentrifugieren erhaltenen Überstand $(20 \mathrm{ml} \triangleq 400 \mu l$ Vollblut), so läßt sich bei Kenntnis des Hämatokritwertes die Acetylcholinesterase-Aktivität für Vollblut $\left(\mathrm{Akt}_{\mathrm{v}}\right)$, Plasma $\left(A k t_{p}\right)$ und Erythrocyten (Akt $)$ berechnen. Die Angabe der Enzymaktivität erfolgt in $\mu \mathrm{Mol}$ Substrat $\mathrm{m} l^{-1}$ Min. $^{-1}$.

\section{Methodik}

\section{Apparatur}

Der automatische Titrator der Fa. Radiometer, Kopenhagen, besteht aus den folgenden Baueinheiten.

1. $\mathrm{pH}-\mathrm{Me}$ Bgerät PHM 26

2. Steuergerät TTT 11

3. automatische Bürette ABU 1 mit Büretteneinheit B 150, Gesamtvolumen $2,5 \mathrm{ml}$

4. temperiertes Reaktionsgefäß, $50 \mathrm{~m} /$ Inhalt mit Magnetrührer Glaselektrode G 202 C, Kalomelelektrode K 401.

Auf die Verwendung des Schreibers SBR 2 wurde bewußt verzichtet, da bei dieser Art der Registrierung die Auflösung und damit die Genauigkeit der Messung reduziert wird. Der Natronlauge-Verbrauch wurde stattdessen direkt am mechanischen Zählwerk der Bürette abgelesen.

Der Hämatokritwert wurde mit der Mikro-Hämatokritzentrifuge der Fa. Christ bestimmt.

\section{Reagenzien und Lösungen}

Eiclppuffer: $\mathrm{pH} 7,383$ bei $37^{\circ}$, Radiometer-Vorschrift: $1,816 \mathrm{~g}$ $\mathrm{KH}_{2} \mathrm{PO}_{4}, 9,501 \mathrm{~g} \mathrm{Na} 2 \mathrm{HPO}_{4} \cdot 2 \mathrm{H}_{2} \mathrm{O}, 1000 \mathrm{~g}$ bidest. Wasser, ausgekocht.

Salzlostung: $0,15 \mathrm{M} \mathrm{NaCl}, 10 \mathrm{~mm} \mathrm{MgCl}_{2} \cdot 17,532 \mathrm{~g} \mathrm{NaCl}, 4,066 \mathrm{~g}$ $\mathrm{MgCl}_{2} \cdot 6 \mathrm{H}_{2} \mathrm{O}$, mit bidest. Wasser auf $2 l$ auffüllen.

Substratlösung: $15 \mathrm{~mm}$ Acetylcholin-jodid. $2,048 \mathrm{~g}$ Acetylcholinjodid mit Salzlösung auf $500 \mathrm{~m} /$ auffüllen. Proben von etwa $6 \mathrm{~m} l$ werden in Reagenzgläser mit Schraubverschluß abgefüllt und in der Tiefkühltruhe eingeftoren.

Natronlange: $0,01 \mathrm{~N}$.

Als Stammlösung wird eine $0,1 \mathrm{~N} \mathrm{NaOH}$ (Fixanal) verwendet, die $1: 10$ mit ausgekochtem bidest. Wasser verdünnt wird.

$1 \mu l \bumpeq 0,01 \mu \mathrm{Mol} \mathrm{NaOH}$.

\section{Versuchsbedingungen}

Die Reaktionsbedingungen sind so ausgelegt, daß die Reaktion nach pseudo-nullter Ordnung abläuft. $\mathrm{pH} 7,40,37^{\circ}, 3 \mathrm{~mm}$ Acetylcholin-jodid, $0,15 \mathrm{M} \mathrm{NaCl}$, $10 \mathrm{~mm} \mathrm{MgCl}$, Stickstoffspülung: $50 \mathrm{~m} / / \mathrm{Min}$., Proportionalband: 0,5 .

Durchführung der Aktivitätsbestimmung Abbildung 1 gibt eine schematische Darstellung des Versuchsablaufs.

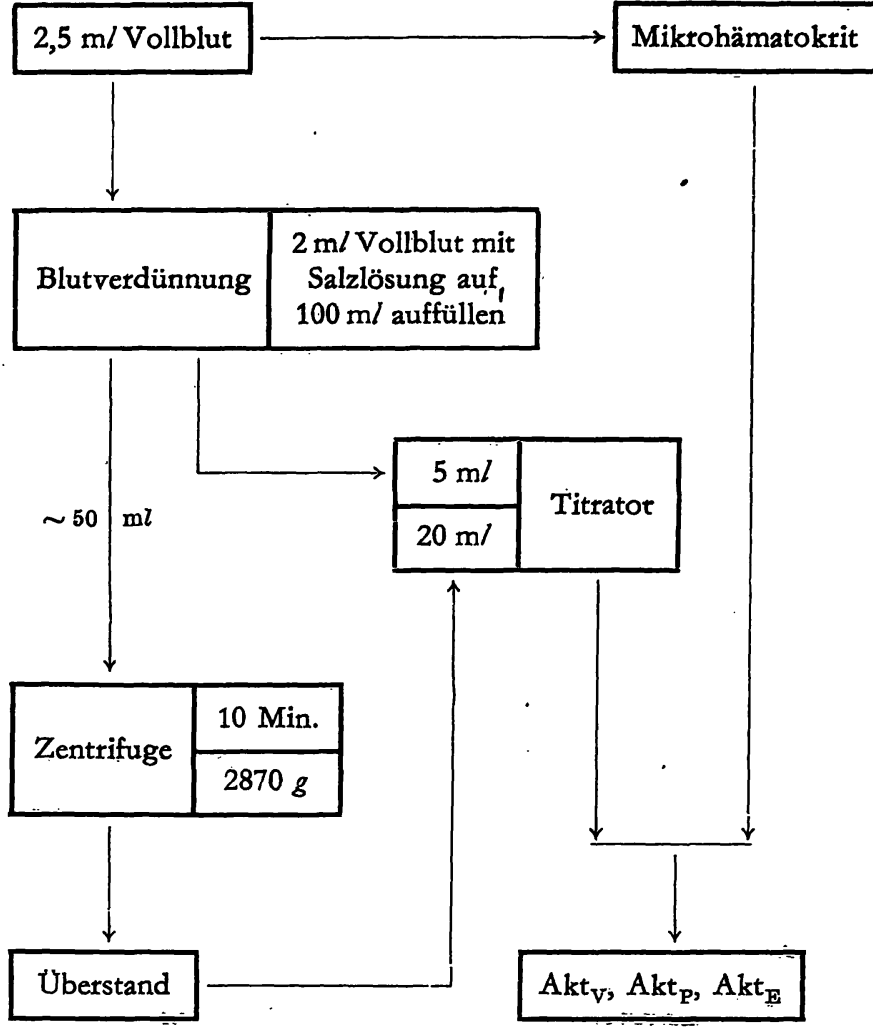

Abb. 1

Arbeitsschema für die Aktivitätsbestimmung

Zur Aktivitätsbestimmung werden $2 \mathrm{~m} l$ heparinisiertes Vollblut im Meßkolben mit Salzlösung auf $100 \mathrm{~m} l$ aufgefüllt. Etwa $50 \mathrm{~m} l$ werden zur Gewinnung des erythrocytenfreien Überstandes zentrifugiert, der Rest findet bei der Bestimmung der Vollblut-Aktivität Verwendung. Die in der Arbeitsvorschrift angegebenen Einstellzeiten dienen der Einstellung der experimentellen Parameter wie Temperatur, $\mathrm{pH}$-Wert, Rührgeschwindigkeit, Stickstoffspülung usw. Um sicher zu sein, daß durch unerwünschte Nebenreaktionen keine Natronlauge verbraucht wird, wird vor der Substratzugabe eine Kontrollmessung ausgeführt.

\section{Vollblut: $\quad 15 \mathrm{~m} /$ Salzlösung \\ $5 \mathrm{ml}$ Blutverdünnung \\ 2 Min. Einstellzeit \\ 3 Min. Kontrollmessung \\ $5 \mathrm{~m} l$ Substratlösung \\ 2 Min. Einstellzeit \\ 5 Min. Aktivitätsmessung \\ Plasma: $\quad 20 \mathrm{~m} l$ Überstand \\ 2 Min. Einstellzeit \\ 2 Min. Kontrollmessung \\ $5 \mathrm{~m} l$ Substratlösung \\ 2 Min. Einstellzeit \\ 5 Min. Aktivitätsmessung}

Das Titrationsergebnis wird in $\mu \mathrm{Mol} \mathrm{NaOH} / \mathrm{Min}$. angegeben, wobei direkt mit dem 5 Minuten-Wert gerechnet wird. Die Berechnung der Differenzen für die 1 Minuten-Intervalle dient lediglich der Kontrolle. 
Beispiel einer Doppelbestimmung: Versuchsperson MS (Tab. 1).

Tab. 1

Wertetabelle für eine Doppelbestimmung von Acetylcholinesterase im Vollblut bzw. Plasma Hämatokrit: 40,$0 ; 40,0 \%$

E Einstellzeit Meßzeit

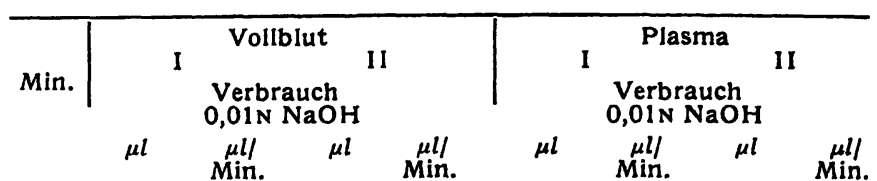

\begin{tabular}{|c|c|c|c|c|c|c|c|c|}
\hline 0 & 0 & 36 & 0 & 31 & 0 & 0 & \multirow{2}{*}{\multicolumn{2}{|c|}{0}} \\
\hline 1 & 36 & \multirow{2}{*}{7} & 31 & \multirow{2}{*}{11} & 0 & \multirow{2}{*}{0} & & 0 \\
\hline 2 & 43 & & 42 & & 0 & & 0 & 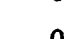 \\
\hline 3 & 46 & 3 & 48 & 8 & 0 & 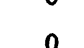 & 0 & 0 \\
\hline 4 & 46 & 0 & 49 & 0 & 0 & 10 & 0 & 36 \\
\hline 5 & 46 & 57 & 49 & 64 & 10 & 46 & 36 & 41 \\
\hline 6 & 103 & \multirow{2}{*}{60} & 113 & 59 & 56 & 40 & 77 & 40 \\
\hline 7 & 163 & & 172 & 59 & 96 & 40 & 117 & 40 \\
\hline 8 & 221 & $\begin{array}{l}58 \\
59\end{array}$ & 231 & 5 & 136 & 41 & 157 & 40 \\
\hline 9 & 280 & 57 & 290 & 59 & 177 & 39 & 197 & 39 \\
\hline 10 & 337 & 5 & 349 & 58 & 216 & 4 & 236 & 38 \\
\hline 11 & 394 & \multirow{2}{*}{$\begin{array}{l}57 \\
57\end{array}$} & 407 & 58 & 256 & & 274 & \\
\hline 12 & 451 & & 465 & & & & & \\
\hline \multicolumn{2}{|c|}{$\begin{array}{l}\mu l / \operatorname{Min}(\bar{x}) \\
\mu \text { ol } / \operatorname{Min}\end{array}$} & $\begin{array}{c}57,6 \\
0,576\end{array}$ & & $\begin{array}{l}58,6 \\
0,586\end{array}$ & & $\begin{array}{r}40,0 \\
0,400\end{array}$ & & $\begin{array}{r}39,4 \\
0,394\end{array}$ \\
\hline
\end{tabular}

\section{Berechnung der Enzymaktivität}

Die Enzymaktivität wird in $\mu \mathrm{Mol}$ Acetylcholin $\mathrm{m}^{-1}$ Min. ${ }^{-1}$ angegeben. Für die Berechnung gilt:

\section{Meßwert = Titrationsergebnis - Blindwert}

Der Blindwert (Spontanhydrolyse des Substrats) beträgt bei dem oben erwähnten Beispiel 0,01 $\mu \mathrm{Mol} / \mathrm{Min}$. Der Hämatokritwert wird als Dezimalbruch eingesetzt. Also 0,40 anstatt $40 \%$.

$$
\begin{aligned}
\mathrm{Akt}_{\mathrm{v}} & =\mathrm{Meßwert}_{\mathrm{v}} \times 10 \\
\mathrm{Akt}_{\mathrm{P}} & =\frac{\text { Meßwert }_{\mathrm{P}} \times 2,5}{1-\text { Hämatokritwert }_{\text {äm }}} \\
\mathrm{Akt}_{\mathrm{E}} & =\frac{\mathrm{Aktv}-(\mathrm{Meßwert} \mathrm{P} \times 2,5)}{\text { Hämatokritwert }}
\end{aligned}
$$

Für das oben angeführte Beispiel folgt:

$$
\begin{aligned}
& \mathrm{Akt}_{\mathrm{v}}=5,71 \mu \mathrm{Mol} \text { Acetylcholin } \mathrm{m} l^{-1} \mathrm{Min}^{-1} \\
& \mathrm{Akt}_{\mathrm{P}}=1,61 \mu \mathrm{Mol} \text { Acetylcholin } \mathrm{m} l^{-1} \mathrm{Min}^{-1} \\
& \mathrm{Akt}_{\mathrm{E}}=11,85 \mu \mathrm{Mol} \text { Acetylcholin } \mathrm{m} l^{-1} \mathrm{Min}^{-1} .
\end{aligned}
$$

\section{Statistik}

Um die Präzision der Methode zu überprüfen, wurden 5 Doppelbestimmungen einer Blutkonserve ausgeführt, deren Ergebnisse in Tabelle 2 zusammengefạt sind.

Die Normalwerte für 20 gesunde Versuchspersonen sind in Tabelle 3 aufgeführt. Zum Vergleich wurde in 6 Fällen die Acetylcholinesterase-Aktivität für eine Plasmaprobe, die durch Zentrifugieren des nicht verdünnten Blutes gewonnen wurde, bestimmt ( $\mathrm{Akt}_{\mathrm{P}}$ ) direkt).
Tab. 2

Kontrolle der Präzision (5 Doppelbestimmungen) AcetylcholinesteraseAktivität in $\mu$ Mol Acetylcholin m $l^{-1} \mathrm{Min}^{-1}$

\begin{tabular}{|c|c|c|c|c|c|}
\hline Vp. & & Akt v & Akt $_{P}$ & Akt $_{E}$ & Akt $_{P}$ direkt \\
\hline GK & $q$ & 6,41 & 1,97 & 13,08 & 1,99 \\
\hline BV & 우 & 7,20 & 1,76 & 13,84 & 1,77 \\
\hline UP & q & 8,03 & 2,37 & 16,00 & 2,41 \\
\hline$M N$ & $0^{\circ}$ & 7,78 & 2,25 & 14,40 & 2,30 \\
\hline JA & $0^{\prime \prime}$ & 8,27 & 2,34 & 15,10 & 2,41 \\
\hline HS & $0^{\circ}$ & 7,99 & 2,14 & 13,50 & 2,22 \\
\hline MK & $0^{\circ}$ & 7,03 & 2,18 & 12,60 & \\
\hline LK & $\sigma^{\prime}$ & 6,84 & 2,44 & 12,00 & \\
\hline GB & $\sigma^{\prime \prime}$ & 7,56 & 1,70 & 13,18 & \\
\hline UP & $0^{\prime}$ & 5,97 & 1,98 & 11,05 & \\
\hline WP & $0^{\circ}$ & 6,36 & 1,90 & 10,82 & \\
\hline $\mathrm{DB}$ & 웅 & 5,95 & 1,51 & 11,59 & \\
\hline KS & 웅 & 8,16 & 2,35 & 16,36 & \\
\hline CC & $0^{\circ}$ & 6,24 & 1,77 & 12,95 & $\cdot$ \\
\hline FV & $\sigma^{\circ}$ & 6,01 & 1,94 & 11,62 & \\
\hline SL & $0^{\circ}$ & 6,82 & 2,39 & 11,91 & \\
\hline MS & Q & 5,71 & 1,61 & 11,85 & \\
\hline GG & \% & 7,09 & 1,73 & 14,48 & \\
\hline EK & $\sigma^{\circ}$ & 7,83 & 1,58 & 14,87 & \\
\hline SG & 우 & 5,28 & 1,20 & 11,40 & \\
\hline$\overline{\mathbf{x}}$ & & 6,93 & 1,96 & 13,13 & \\
\hline $\mathbf{s}$ & & 0,91 & 0,35 & 1,64 & \\
\hline VK & & $13,1 \%$ & $17,8 \%$ & $12,6 \%$ & \\
\hline
\end{tabular}

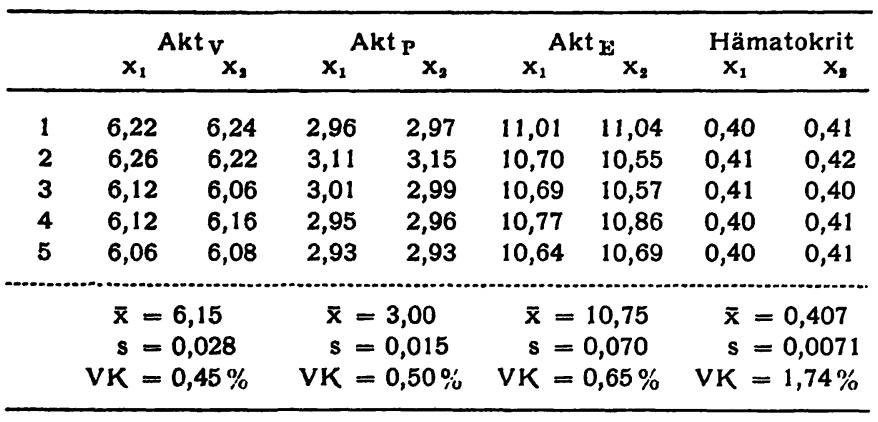

Tab. 3

Normalwerte erwachsener Versuchspersonen. AcetylcholinesteraseAktivität in $\mu \mathrm{Mol}$ Acetylcholin $\mathrm{ml}^{-1} \mathrm{Min}^{-1}$ bei $\mathrm{pH} 7,40,37^{\circ}, 3 \mathrm{~mm}$ Acetylcholin

\section{Diskussion}

Die beschriebene Methodik erlaubt eine schnelle und exakte Bestimmung der Acetylcholinesterase-Aktivität in Vollblut, Plasma und Erythrocyten. $\mathrm{Da}$ grundsätzlich große Volumina pipettiert werden, ist der Pipettierfehler vernachlässigbar klein. Die Variationskoeffizienten von $0,45,0,49$ und $0,65 \%$ weisen auf eine hohe Präzision hin. Lediglich die Hämatokritbestimmung zeigt einen Variationskoeffizienten von $1,74 \%$.

Auf die Hämolyse wurde verzichtet, da zwischen der Aktivität hämolysierter und nicht hämolysierter Proben kein signifikanter Unterschied festgestellt werden konnte. Steht zu wenig Blut zur Verfügung, so kann ohne weiteres $1 \mathrm{~m} l$ Vollblut auf $50 \mathrm{~m} l$ verdünnt werden, wobei man auf die Doppelbestimmung verzichtet. Wie Tabelle 1 zeigt, liegen die Ergebnisse bei Doppelbestimmungen so dicht beisammen, daß dieses Vorgehen durchaus gerechtfertigt ist. Eine Anpassung der Methode zur Bestimmung der Acetylcholinesterase-Aktivität im Tierblut ist leicht möglich. 
Der Vergleich mit Normalwerten anderer Autoren ist im Augenblick nicht möglich, da die hier gezeigte Differenzierung in Vollblut, Plasma und Erythrocyten unter vergleichbaren methodischen Bedingungèn $(\mathrm{pH}-$ Wert, Temperatur, Substratkonzentration) bisher noch nicht durchgeführt worden ist.

Gute Übereinstimmung unserer Werte mit den Angaben von Dabeiv (12) ergab sich hinsichtlich der Acetyl-
cholinesterase-Aktivität im Plasma bei 3 Kindern unter 6 Jahren (nicht in Tabelle 2 enthalten).

Unter Zugrundelegung der in Tabelle 2 aufgeführten Werte ergeben sich für $\overline{\mathrm{x}} \pm 2 \mathrm{~s}$ die folgenden Bereiche:

$\mathrm{Akt}_{\mathrm{v}}: 5,11-8,75 \mu \mathrm{Mol}$ Acetylcholin $\mathrm{m} l^{-1} \mathrm{Min.}^{-1}$

$\mathrm{Akt}_{\mathrm{P}}: 1,26-2,66 \mu \mathrm{Mol}$ Acetylcholin $\mathrm{m}^{-1} \mathrm{Min}^{-1}$

$\mathrm{Akt}_{\mathrm{E}}:$ 9,85-16,41 $\mu \mathrm{Mol}$ Acetylcholin $\mathrm{m} l^{-1} \mathrm{Min}^{-\mathbf{1}}$.

\section{Literatur}

1. Nenner, M. und W. D. ERdManN, Dtsch. med. Wschr. 94, 504 (1969). - 2. Hafn, H. L. und D. Henschler, Atch. Toxikol. 24, 147 (1969). - 3. KNolle, J., Arch. Toxikol. 26, 29 (1970). 4. Ammon, R., Pflügers Arch. ges. Physiol. 233, 486 (1934). 5. Witter, R. F., Tox. Appl. Pharmacol. 4, 313 (1962). - 6. Michel, H. O., J. Laborat. clin. Med. S. Louis 34, 1564 (1949). -
7. Kalow, W. und K. Genest, Canad. J. Biochem. 35, 339 (1957). 8. Weber, H., Dtsch. med. Wschr. 91, 1927 (1966). - 9. KNedeL, M. und R. Bötrger, Klin. Wschr. 45, 325 (1967). - 10. GrICK, D., Biochem. J. 31, 521 (1937). - 11. Jörgensen, K., Scand. J. Clin. Laborat. Invest. 11, 282 (1959). - 12. DABEw, D., diese Z. 8,12 (1970).

Dr. M. Nenner 34 Göttingen Geiststr. 9 